\title{
Buddy-to-Buddy, a citizen soldier peer support program to counteract stigma, PTSD, depression, and suicide
}

\author{
John F. Greden, ${ }^{1,3}$ Marcia Valenstein, ${ }^{1,2}$ Jane Spinner, ${ }^{1}$ Adrian Blow, ${ }^{4}$ Lisa A. Gorman, ${ }^{4}$ \\ Gregory W. Dalack, ${ }^{1}$ Sheila Marcus, ${ }^{1}$ and Michelle Kees ${ }^{1}$ \\ ${ }^{1}$ University of Michigan Department of Psychiatry and Comprehensive Depression Center, University of Michigan, Ann Arbor, \\ Michigan. ${ }^{2}$ Ann Arbor Veterans Administration Hospital, University of Michigan, Ann Arbor, Michigan. ${ }^{3}$ Molecular and \\ Behavioral Neurosciences Institute, University of Michigan, Ann Arbor, Michigan. ${ }^{4}$ Michigan State University, Lansing, \\ Michigan
}

Address for correspondence: John F. Greden, M.D., University of Michigan Health System, 4250 Plymouth Road, Ann Arbor, Michigan 48109. gredenj@umich.edu

Citizen soldiers (National Guard and Reserves) represent approximately $40 \%$ of the two million armed forces deployed to Afghanistan and Iraq. Twenty-five to forty percent of them develop PTSD, clinical depression, sleep disturbances, or suicidal thoughts. Upon returning home, many encounter additional stresses and hurdles to obtaining care: specifically, many civilian communities lack military medical/psychiatric facilities; financial, job, home, and relationship stresses have evolved or have been exacerbated during deployment; uncertainty has increased related to future deployment; there is loss of contact with military peers; and there is reluctance to recognize and acknowledge mental health needs that interfere with treatment entry and adherence. Approximately half of those needing help are not receiving it. To address this constellation of issues, a private-public partnership was formed under the auspices of the Welcome Back Veterans Initiative. In Michigan, the Army National Guard teamed with the University of Michigan and Michigan State University to develop innovative peer-to-peer programs for soldiers (Buddy-to-Buddy) and augmented programs for military families. Goals are to improve treatment entry, adherence, clinical outcomes, and to reduce suicides. This manuscript describes training approaches, preliminary results, and explores future national dissemination.

Keywords: citizen soldiers; peer-to-peer; PTSD; depression; suicide

\section{Introduction}

Forty percent or more of the approximate two million troops that have been deployed to military conflicts in Afghanistan and Iraq have been members of America's National Guard or military Reserve units. ${ }^{1}$ These "citizen soldiers" experience traumas and stresses comparable to those encountered by active duty soldiers, such as battlefield conflicts and injuries; improvised explosive device explosions; deaths among fellow soldiers in their units; "downrange funerals" (a colloquial term used by some deployed military soldiers); and prolonged separation from loved ones.

The clinical and social consequences of these experiences include posttraumatic stress disorder (PTSD) symptoms, clinical depression, sleep dysregulation and nightmares, self-medication, sub- stance use and abuse, and suicide thoughts, acts, and occasional tragic deaths by suicide. For some, co-occurring traumatic brain injuries confound the clinical presentation. $^{2-5}$

Military personnel experience major psychiatric disorders at rates comparable to the general population, but these rates increase following deployment. ${ }^{6,7}$ In one study, combat experience during Operation Enduring Freedom (OEF) and Operation Iraqi Freedom (OIF) was significantly associated with use of mental health services and military attrition following deployment. ${ }^{6}$ Forty-two percent of citizen soldiers from Reserve and National Guard units report mental health issues suggesting the need for evaluation and possible treatment; yet, many do not initiate treatment. ${ }^{5,8}$ Only $54 \%$ of soldiers referred through the Post Deployment Health Assessment screening process subsequently followed 
through with a mental health visit, ${ }^{6}$ and only $30 \%$ with identified need reported receiving minimally adequate treatment (adequate medication trial or at least eight psychotherapy sessions). ${ }^{5}$

Although research is needed to improve effectiveness of available treatments, innovative programs to overcome stigma and associated barriers to treatment entry and adherence are arguably as crucial.

\section{Unique stresses and barriers to treatment entry and adherence among citizen soldiers}

Citizen soldiers commonly encounter additional stressors or barriers that are different from active duty soldiers and interfere with entry and adherence into treatment. Perhaps most important is the inexorable stigma associated with seeking care. Although a common barrier to active duty soldiers as well, stigma may be even more difficult to overcome in community settings. Other postdeployment stresses include financial pressures and for some, income reductions; concerns about job availability or job security upon returning from deployment; home foreclosures; future prospects of being deployed; and absence of the readily available medical and psychiatric facilities that would have been available had they returned to an active duty military post. For those in rural areas, clinical services sometimes are available only at great distances, generating long travel times that make appointments difficult to keep. Finally, the dispersal and separation across large state regions and loss of everyday contact with military buddies mean that citizen soldiers do not have colleagues and comrades readily available for valuable sharing of experiences and support. A key consequence is that many of those needing professional treatment are reluctant, and far less likely to receive it unless these barriers are overcome; without treatment, clinical and functional deterioration is more likely.

This report does not focus upon specific treatment interventions for PTSD, depression, suicide, or their co-occurrence. Available treatments are described in detail elsewhere and extensive Department of Defense projects are under way to improve available treatments. ${ }^{9}$ Instead, we aim to describe refinements and innovations in peer-to-peer strategies to help counter the unique barriers faced by citizen soldiers to aid them in overcoming stigma and promote essential entry into and adherence with appropriate treatments.

\section{Michigan Army National Guard: a prototype of citizen soldier in America}

The Michigan Army National Guard's (MI ARNG) experiences illustrate the growing importance of citizen soldiers in ongoing military conflicts. Since 2001 , around $90 \%$ of the approximate 9,000 members of the MI ARNG have deployed to Afghanistan and/or Iraq, many on multiple occasions. Citizen soldiers, as already summarized, manifest symptoms compatible with PTSD, depression, substance use, and interpersonal conflicts to a greater degree as active duty soldiers. Such symptoms are sometimes not evident until after significant time delays. Some returning soldiers also have manifestations of traumatic brain injury, sometimes referred to as a new signature injury in the OEF/OIF conflict. ${ }^{2,5}$ To help respond to these concerns and proactively address these "silent injuries," MI ARNG leaders forged collaborations with faculty and staff members of Michigan State University and the University of Michigan; steps involved in forging such collaborations are described in a separate report. ${ }^{9}$

A survey tool was used to understand the scope of veterans' problems and mental health needs. This survey was completed by 926 returning MI ARNG soldiers and spouses. Approximately, $40 \%$ of this sample screened positive for a mental health problem of some kind translating to approximately 3,500 of the 9,000 MI ARNG soldier force. Approximately, $8 \%$ of those assessed for a mental health problem reported suicidal thoughts (Table 1 ).

A crucial issue was identified: only $47 \%$ of returned citizen soldiers with reported clinical problems had sought any help. The most common reported reasons for not seeking help were linked to

Table 1. Reported reasons for not seeking help

- Do not want it in military records (27\%)

- Unit leadership might treat me differently (20\%)

- Too embarrassing (17\%)

- Harm career (17\%)

- Costs (15\%)

- Do not know where to go to get help (6\%)

- No providers in my community $(6 \%)$

- Transportation (5\%) 


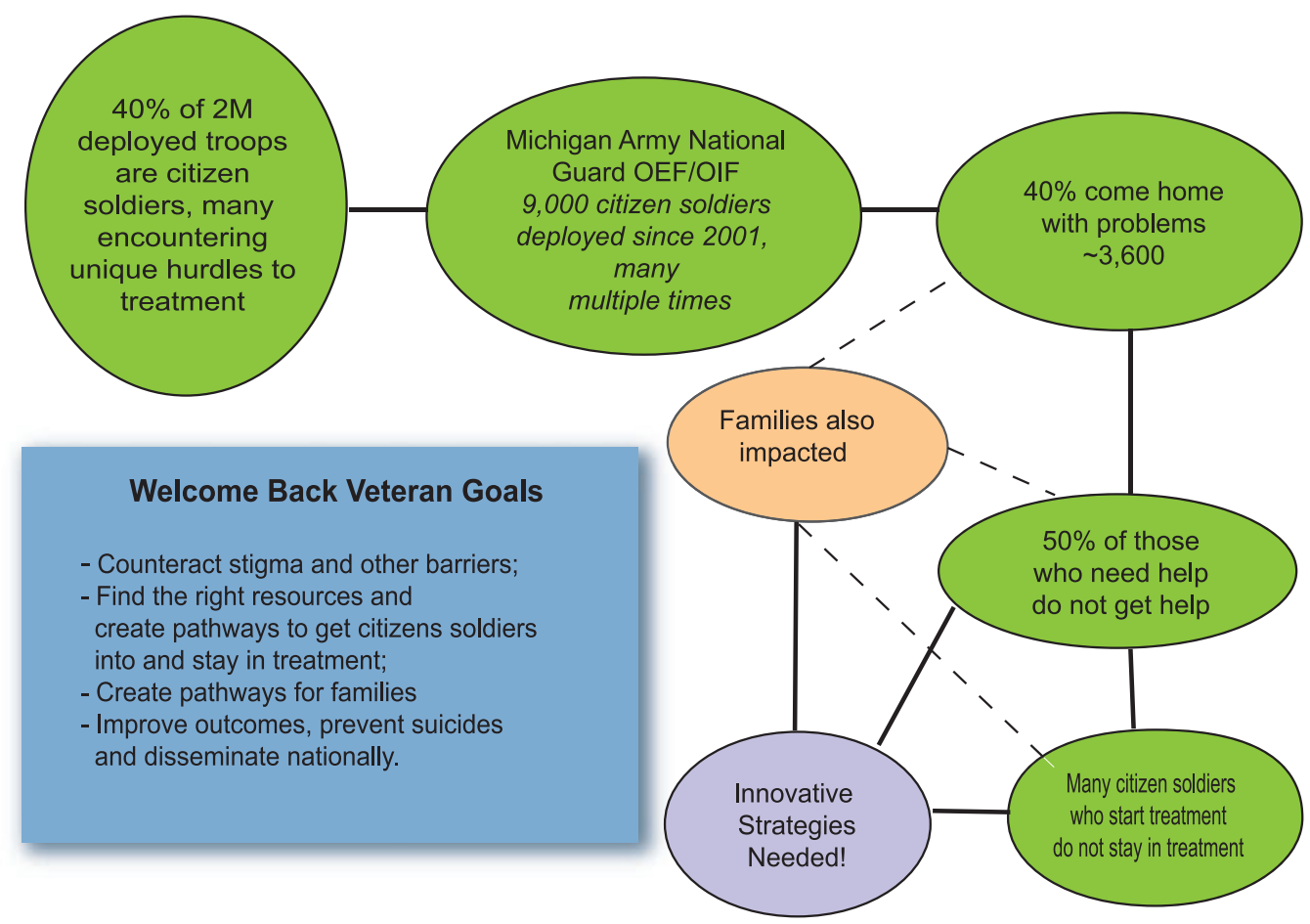

Figure 1. Scope of problems among citizen soldiers and families: need for innovative peer-to-peer programs and national dissemination strategies. (In color in Annals online.)

stigma, fear of being seen as weak, concerns about confidentiality of military records, fears about damaging their future careers, and for a much smaller percentage, uncertainty about where to go for treatment or practical barriers in getting there.

Efforts to counteract these stigma barriers appear fundamental if we are to successfully identify and treat PTSD, depression, suicide, and related problems among returning veterans. Treatments can not work unless they are delivered; innovative strategies are required. A summary of the issues is portrayed in Figure 1.

\section{Welcome Back Veterans: a private-academic-military partnership to address mental health problems of returning veterans}

Responding to the well-reported problems of returning military veterans nationally, start-up philanthropic funding was provided in a national initiative known as "Welcome Back Veterans" (WBV) (www.welcomebackveterans.org). WBV leadership worked in partnership with Major League Baseball, the McCormick Foundation, and initially the
Ad Council to generate funding. The University of Michigan, Weill-Cornell, and Stanford University were selected as three "WBV core centers" to help mobilize clinical outreach and national dissemination efforts to address some of the unmet needs of returning veterans. The aims were to integrate and disseminate gains and specialty knowledge from each university, coordinate with advances occurring elsewhere, and augment the veterans administration programs available to returning veterans. A University of Michigan team and a Michigan State University team collaborated with the leadership of the MI ARNG to design and implement an innovative peerto-peer program for returning soldiers. Although not the focus of this report, the University of Michigan team also forged accompanying strategies to reach out to families and children of MI ARNG citizen soldiers.

\section{Buddy-to-Buddy: a peer-to-peer program for returning citizen soldiers}

The collaborative team hypothesized that the crucial steps of counteracting stigma and improving treatment entry and adherence might best be enhanced 
by "using culture to change culture." When discussing their OEF/OIF deployments, many soldiers conveyed, that "if you haven't been there, you don't get it," "we believe in taking care of our own," and "other veterans can be trusted." For many, the nation's established system of medical and psychiatric programs and traditional clinical teams of medical, psychology, or social work leaders are not necessarily starting points. Correlative comments occasionally conveyed optimism, such as "another veteran who has been there may make it easier to get help." A logical extension of these observations led to the realization that when cultural barriers impeded treatment entry or adherence, peer-to-peer influences may be a crucial cultural starting point in overcoming them. ${ }^{10}$

For these reasons, university partners worked with the MI ARNG leaders and staff to develop what became known as "Buddy-to-Buddy." More detailed descriptions of Buddy-to-Buddy will be described in forthcoming publications. This manuscript aims to summarize Buddy-to-Buddy concepts, goals, acceptance, and dissemination to date; future steps required to confirm the clinical effectiveness of peerto-peer strategies; and future recommendations as to how this and other related programs may play crucial roles for returning veteran populations. We also hypothesize that these initiatives may be a valuable adjunct and foundation in efforts to reduce veteran suicides.

\section{Buddy-to-Buddy components, goals, principles, and philosophies: a brief summary}

Buddy-to-Buddy ensures contact with every returning MI ARNG soldier by using soldier peers. Trained peers regularly contact their assigned panel of soldiers to "check in," help identify those with clinical needs, encourage registration and entry into Veterans Administration Hospital (VAH) or military programs, and develop strategies to enhance enrollment in community treatment programs that are perceived as safe and acceptable should other alternatives be unworkable or unacceptable. In addition, soldier peers support adherence after starting treatment.

The program also seeks to identify and train selected community clinicians, orienting them to military culture and combat issues. This serves to enhance the resource pool and optimizes the like- lihood for readily available interventions. These efforts build on earlier efforts initiated by Michigan State University and MI ARNG to emphasize collaboration with an array of quality providers, because community resources and a geographically dispersed network of clinical providers are essential to address the approximately $50 \%$ of citizen soldiers that need and would benefit from clinical intervention but are not currently receiving it. More such efforts are planned.

Consistent with military traditions, "buddies, families, and resiliency" became constant messages, accompanied by the messages of "you are not alone, treatment works, it has helped many of your buddies, and pursuing help is a sign of strength.” Another major thrust sought to link veterans with other concrete resources they need to reduce stressors, such as employment benefits, housing, and financial guidance.

\section{Creating and training the pool of Buddy Ones and Buddy Twos}

A starting principle of the Buddy-to-Buddy outreach program was to ensure that all soldiers were contacted, not just the ones who were exhibiting clear and unmistakable signs of needing help. A twotier program was created. The first tier of peer-topeer, called Buddy Ones, consists of soldiers within each National Guard unit. The recruitment, operation, and oversight are provided by the MI ARNG. A second, smaller tier, called Buddy Twos or Volunteer Veterans, is operated by veterans outside of the Guard, and is overseen by University of Michigan staff.

"Buddy-One" individuals were identified for further training by their Chain of Command. They were chosen either by their position in the chain of command or because they were informal leaders of their units, depending on the preferences of the Command of each specific Battalion. Care was taken that peers supporters and their soldiers did not have great disparity in rank.

Training for Buddy Ones approximates $3 \mathrm{~h}$ and conveys the program rationale and philosophy, roles, communications skills, including what to do in case of emergencies, and an overview of resources of all types. A manual and quick reference cards were prepared and are distributed to Buddy One personnel. These are available online or upon request. Support and retraining are provided during 
drill weekends and ongoing consultation continues to be integral to refinements.

"Check-in" calls are incorporated into the design. A strong message for Buddy One personnel is that "your job is not to give help, it's to get help." Special strategies emphasize earlier identification of worrisome behavior, believing that those who know the soldier can assess whether something is wrong; legitimize seeking help; communicate knowledge of referral sources; and follow-up to aid adherence. Buddy Twos are veterans from outside the Guard who serve as back up for the Buddy Ones. They receive more intensive training in communication skills, including motivational interviewing, and become well-versed in both military and community resources that they can use as needed. The Buddy Twos staff the National Guard Armories statewide during drill weekends and serve as on-site resources to address concerns. Buddy Twos often help individuals navigate systems, such as the Veterans Administration in order to facilitate access. To date, approximately 350 Buddy Ones and 32 Buddy Twos have been trained.

Importantly, MI ARNG command leaders have been invested in identifying and supporting citizen soldiers with these "invisible injuries." Their leadership and partnership have been essential. Individual soldiers identified as potential Buddy Ones or Buddy Twos have been similarly invested, helpful, and committed to helping their returning veteran colleagues; their participation and suggestions were instrumental in planning, shaping, and implementing the program as well as suggesting modifications.

\section{Buddy-to-Buddy: preliminary observations and evaluations}

Among the Buddy-to-Buddy participants surveyed, 9 of 10 understand the intent of the program; approximately two-thirds are receiving regular calls from their Buddy and feel comfortable talking with their Buddy. More than half reported using resources or services suggested by their Buddy. In many cases, these were referrals for concrete resources, such as assistance with benefits, jobplacement services, financial assistance, or legal help. Of primary relevance to this report, more than $20 \%$ have been referred to formal treatment by their Buddy, reflecting previously unmet clinical needs. These referrals have been made to VAHs and Vet Centers, community-based mental health providers, military-sponsored agencies, such as Military One Source, and to community clinical resources. These referrals are in the process of being evaluated in greater detail and longer-term outcome evaluations are being proposed.

MI ARNG leaders and participants have endorsed these initiatives, conveying that they merge favorably with the fundamentals of National Guard culture. Predictably, not all returning citizen soldiers are likely to be responsive to peer-to-peer initiatives, but clearly more of those in need of treatment are being reached by this use of buddy culture. Even more would be reached with extensions of the program. Synergy with adjunct innovative strategies may be required to reach those not yet receptive to the Buddy-to-Buddy approach. Examples include family outreach programs, incorporation of respected speakers to help counteract stigma, and others.

\section{Potential role of peer-to-peer programs in reduction of suicides among citizen soldiers}

Rates of suicide in active military personnel have been increasing since 2003 and now surpass age- and gender-matched nonveterans. These increases have led to a fervent search for new interventions. ${ }^{11-14}$ In a recent Department of Defense and Veterans Administration Conference on Suicide Prevention among Veterans, Shinsheki conveyed that of the approximate 85 deaths a day by suicide in America, about 18 are by veterans and most are not currently receiving treatment in the $\mathrm{VAH} .{ }^{15}$

It is our hypothesis that the Buddy-to-Buddy program has strong potential to augment suicide prevention programs by applying the previously described, that is, the "use of culture to change the culture of treatment avoidance." Strategies would emphasize earlier identification of worrisome, threatening or self-injurious behaviors, knowing about referral sources, "helping show the way," and longer-term support of adherence to treatment once started. ${ }^{16}$

Determining whether suicide prevention efforts among returning soldiers are effective requires close attention to co-occurring diagnoses or comorbidities. PTSD and clinical depression commonly coexist among returning soldiers. ${ }^{17,18}$ Stresses during and after deployment play a role in both, and may precipitate both PTSD and/or major depressive disorder (MDD). MDD is the diagnosis most closely 
associated with suicide and its primary ages of onset (15-24 years of age) overlap closely with the age range for most soldiers and returning veterans. ${ }^{19-21}$ In the general population, $80 \%$ or more of those who die by suicide are struggling with $\mathrm{MDD}^{19}$; yet, depression often remains undiagnosed in all populations. ${ }^{22}$ Reasons are multiple: depressive symptoms may be mild and sporadic in early stages so they may be overlooked or attributed exclusively to external stresses. In some cases, symptoms may be clouded or "hidden" by co-occurring diagnoses. PTSD, for example, may have greater cultural acceptance in military populations than a diagnosis of depression, although this has not been adequately studied. Unfortunately, co-occurring clinical depression, when untreated, routinely evolves over subsequent years into an episodic, recurrent, worsening chronic illness that becomes more difficult to treat. ${ }^{22}$

Because co-occurrence is the norm, concomitant treatment of all prevailing clinical syndromes should be the norm. Treatment of the individual citizen soldiers should be as comprehensive as possible, addressing all existing contributors. This includes PTSD, depression, sleep dysregulation, and substance misuse.

Another potential rationale for developing Buddy-to-Buddy type programs to aid suicide prevention is that the goals, culture, and strategies are parallel to those emphasized by suicide prevention programs encountered by veterans during their military deployments. Examples are ACE (Ask, Care, Escort), ${ }^{23}$ ACT (Ask, Care, Treat), ${ }^{24}$ and R.A.C.E. (Recognize, Ask, Care, Escort $)^{25} \cdot{ }^{10}$

In essence, we hypothesize that coupling Buddyto-Buddy or comparable documented peer support programs with evidence-based treatment interventions would help address many untreated individuals; improve entry and adherence into treatment; accelerate clinical improvements by enabling exposure to effective treatments for PTSD, depression and other risk variables; and hopefully reduce suicides for returning veterans. Lessons learned to date are summarized in Table 2.

\section{Augmenting strategies: family outreach and use of spokespersons}

Survey data indicate high levels of parenting and marital stress at the time of reintegration. In addition, spouses uniformly report a desire to have increased support during deployment, suggesting
Table 2. Buddy-to-Buddy program summary highlights

- Similar risks: Citizen soldiers struggle with PTSD, clinical depression, sleep dysregulation, substance use disturbances, and increased risk of suicide upon returning from deployment at rates comparable or greater than active duty soldiers. Comorbidity (co-occurrence of one or more diagnostic syndromes) is the norm, not the exception.

- Unique barriers and stresses: Citizen soldiers encounter additional stresses and unique hurdles to receiving treatment upon returning to their community, including financial and family stresses, stigma, unavailability of treatment resources, and separation from military support systems. Stigma remains formidable.

- Inadequate treatment entry and adherence: Only about half of those in need enter treatment. Overcoming stigma-related barriers is an essential first step for these returning citizen soldiers if clinical help is to be provided and suicides and other problems prevented.

- Prevailing culture is a barrier: Advice to seek treatment often ignored. The prevailing culture remains "If you haven't been there you don't get it."

- "Buddy-to-Buddy:" using culture to change culture: Buddy-to-Buddy uses military culture to change the stigma culture; peer-to-peer appears to be a powerful approach to addressing stigma and associated barriers.

- Goals of Buddy-to-Buddy are to get help, not to give help.

- Private-Public-Academic-Military partnerships are fundamental: When dealing with citizen soldiers in the National Guard and Reserves, collaborations, mutual respect, and understanding are essential. Involvement of military leadership is fundamental at all stages.

- Buddy-to-Buddy may aid suicide prevention: studies are required but treatment entry is essential if treatments are to work.

- Dissemination strategies are needed: Because of national scope, dissemination strategies must be developed. Multiple voices must be recruited to help counteract stigma. Emerging clinical networks are positioned to aid and would greatly accelerate dissemination strategies.

that early interventions both preceding and during deployment may reduce symptom severity in spouses. Such interventions also could target risk factors for parenting and marital strain. These prevention strategies might reduce marital dyadic 
and parenting stress upon reintegration, in turn reducing stresses impacting the soldier. Moreover, providing spouses with resources needed for their own symptoms often serves as a motivation for spouses to encourage their soldier partners toward intervention.

Other trusted spokespersons similarly can be recruited and educated in mental health literacy to help destigmatize these silent brain injuries, and to emphasize the importance of early treatment and maintenance of wellness, support systems, and suicide prevention strategies. They often are equally valuable in helping community members understand the problems being faced by returning veterans. These may be celebrities, athletes, politicians, or local citizens willing to lend their voices to help educate the public while simultaneously decreasing stigma.

\section{Future recommendations}

Citizen soldiers live in coexisting cultures. They are military personnel who spend most of their lives in civilian settings. They have played crucial roles in ongoing military conflicts. During deployment and postdeployment, they experience similar clinical problems to active duty soldiers, but upon returning home often lack the available support systems of military posts. Although Veterans Administration and community resources are available for most, among many there is prevailing reluctance to use them. A major national initiative is needed to enable a far greater percent of returning citizen soldiers with mental health problems to break through their internal resistance, enter, and hopefully benefit from available treatments.

We strongly recommend that Buddy-to-Buddy be thoroughly evaluated for efficacy and that if it is effective in improving soldier outcomes, that it be disseminated nationally, national training programs be launched, efforts be linked with evaluation outcome assessments, and proxy serial evaluation measures be used when seeking to evaluate possible suicide risk. ${ }^{11,21}$ Suicide has a low base rate so truly large samples must be studied, accompanied by serial assessments of suicide ideation, acts, prior history of suicide attempts, and identification of well-documented risk variables, such as depression, PTSD, substance abuse, and sleep dysregulation. Dissemination predictably can be acceler- ated if already existing national networks are incorporated into these academic-military-community partnerships.

We also recommend that flexibility and individual preference be recognized as important variables when seeking to get reluctant individuals into treatment. Stigma promotes strong recalcitrance to treatment, so it may be essential to match referral resources to the veterans' and family members' preferences. Motivational interviewing can be used effectively as an approach to encourage treatment entry. ${ }^{26}$ Finally, to maintain follow-up when geographic barriers exist, telemedicine interventions may be essential. ${ }^{27,28}$

The strategy we are emphasizing uses trusted fellow veterans (buddies) and augmenting supporting personnel as allies to improve treatment entry and adherence. Evidence to date indicates that this approach may help many overcome prevailing stigma. Consistent with both military culture and centuriesold clinical values, the goals are that no citizen soldiers will be left behind and that those struggling with invisible wounds of war will be welcomed back to a healthier future.

\section{Acknowledgments}

The authors acknowledge indispensable contributions from academic colleagues at Michigan State University, the Ann Arbor Veterans Administration Hospital, University of Michigan, in addition to Weill-Cornell and Stanford, the other core centers in the Welcome Back Veterans collaboration. We also acknowledge support from Health Services Research and Development Service, Department of Veterans Affairs. RRP 09-420, Families and Communities Together Coalition, Michigan State University, Department of Human Development and Family Studies and Biomedical Research Informatics Center at Michigan State University. Major League Baseball, the McCormick Foundation, the Meader Research Fund, and the Entertainment Industry Foundation (EIF) provided critical financial support. Finally, and most importantly, the participation of returning citizen soldiers and leaders of the Michigan Army National Guard (MI ARNG) made it possible to develop and steadily refine the Buddy-go-Buddy and family programs.

\section{Conflicts of interest}

The authors declare no conflicts of interest. 


\section{References}

1. Waterhouse, M. \& J. O’Bryant. 2008. National Guard personnel and deployments: fact sheet. Congressional Research Service, The Library of Congress. Order Code RS22451. January.

2. Hoge, C.W., D. McGurk, J.L. Thomas, et al. 2008. Mild traumatic brain injury in U.S. soldiers returning from Iraq. $N$. Engl. J. Med. 358: 453-463.

3. Jacobson, I.G., M.A. Ryan, T.I. Hooper, et al. 2008. Alcohol use and alcohol-related problems before and after military combat deployment. JAMA 300: 663-675.

4. Schneiderman, A., E. Braver \& H. Kang. 2008. Understanding sequelae of injury mechanisms and mild traumatic brain injury incurred during the conflicts in Iraq and Afghanistan: persistent postconcussive symptoms and posttraumatic stress disorder. Am. J. Epidemiol. 167: 14461452.

5. Tanielian, T. \& L.H. Jaycox. eds. 2008. Invisible Wounds of War: Psychological and Cognitive Injuries, Their Consequences, and Services to Assist Recovery. RAND. Santa Monica, CA. iii-iv; 3-7; 123-134.

6. Hoge, C.W., J.L. Auchterlonie \& C.S. Milliken. 2006. Mental health problems, use of mental health services, and attrition from military service after returning from deployment to Iraq or Afghanistan. JAMA 295: 10231032.

7. Riddle, J.R., T.C. Smith, B. Smith, et al. for the Millenium Cohort Study Team. 2007. Millenium cohort: the 2001-2003 baseline prevalence of mental disorders in the U.S. military. J. Clin. Epidemiol. 60: 192-201.

8. Milliken, C.S., J.L. Auchterlonie \& C.W. Hoge. 2007. Longitudinal assessment of mental health problems among active and reserve component soldiers returning from the Iraq war. JAMA 298: 2141-2148.

9. Dalack, G.W., A. Blow, M. Valenstein, et al. 2010. Working together to meet the needs of Army National Guard soldiers: an academic-military partnership. Psychiatric Services Accepted.

10. Emmanuel, L.O. 2009. VA hires vets to go find comrades who need help. Mar 24 2009. Associated Press. http://www. armytimes.com/news/2009/03/ap_va_veterans_outreach_ 032509/(accessed August 26, 2010).

11. Army Suicide Prevention Program (ASPP). 2009. Shoulder to Shoulder: no soldier stands alone. http://www.armyg1. army.mil/hr/suicide/(accessed April 6, 2009).

12. Basu, S. 2009. Suicide rate among soldiers at all-time high. U.S. Medicine. March. http://www.usmedicine.com/ articles/suicide-rate-among-soldiers-at-all-time-high.html (accessed August 17, 2010).

13. Kuehn, B.M. 2009. Soldier suicide rates continue to rise: military, scientists work to stem the tide. JAMA 301: 11111113.

14. US Army(b), Wright, LT COL G., Media Relations Division, OCPA. 2009. Army releases March suicide data. News release. http://www.army.mil/newsreleases/2009/04/10/19537army-releases-march-suicide-data/(accessed April 30, 2009).

15. Shinsheki, E. 2010. Department of Defense/VA Suicide Prevention Conference: Building Strong and Resilient Communities. January 11. Washington, DC. http://wwwl.va.gov/ opa/speeches/2010/10_0111hold.asp (accessed August 17, 2010).

16. Valenstein, M., D. Eisenberg, J.F. McCarthy, et al. 2009. Service implications of providing intensive monitoring during high-risk periods for suicide among VA patients with depression. Psychiatr. Serv. 60: 439-444.

17. Keane, T.M., A.D. Marshall \& C.T. Taft. 2006. Posttraumatic stress disorder: etiology, epidemiology, and treatment outcome. Annu. Rev. Clin. Psychol. 2: 161-197.

18. Kilpatrick, D.G., K.C. Koenen, K.J. Ruggiero, et al. 2007. The serotonin transporter genotype and social support and moderation of posttraumatic stress disorder and depression in hurricane-exposed adults. Am. J. Psychiatry 164: 16931699.

19. Joiner, T.E. 2005. Why People Die by Suicide. Harvard University Press. Cambridge, MA.

20. Oquendo, M.A., D. Currier \& J.J. Mann. 2006. Prospective studies of suicidal behavior in major depressive and bipolar disorders: what is the evidence for predictive risk factors? Acta Psychiatr. Scand. 114: 151-158.

21. Valenstein, M., H.M. Kim, D. Ganoczy, et al. 2009. Higherrisk periods for suicide among VA patients receiving depression treatment: prioritizing suicide prevention efforts. J. Affect Disord. 112: 50-58.

22. Greden, J.F. 2001. The burden of disease for treatmentresistant depression. J. Clin. Psychiatry 62 (Suppl. 16): 26-31.

23. Department of Veterans Affairs. 2009. ACE, suicide prevention for veterans and their families and friends. http://www.mentalhealth.va.gov/docs/VA_Brochure_08_25_ 2009.pdf (accessed August 17, 2010).

24. Bureau of Navy Personnel. Life Counts! ACT. http:// www.npc.navy.mil/NR/rdonlyres/7A8A1BD2-0E80-47788759-779EB66C54E2/0/Suicide_Prevention_Trifold.pdf (accessed August 17, 2010).

25. United States Marine Corps. Never Leave a Marine Behind, R.A.C.E., Suicide Prevention. 2010. http://www. usmc-mccs.org/display_files/R_A_C_E\%20BI-Fold\% 20with\%20Explanation\%20Page.pdf. May 12, 2010 (accessed August 17, 2010).

26. Hettema, J.E., J.M. Steele \& W.R. Miller. 2005. Motivational interviewing. Annu. Rev. Clin. Psychol. 1: 91-111.

27. Ludman, E.J., G.E. Simon, L.C. Grothaus, et al. 2007. A pilot study of telephone care management and structured disease self-management groups for chronic depression. Psychiatr. Serv. 58: 1065-1072.

28. Hunkeler, E.M., J.F. Meresman, W.A. Hargreaves, et al. 2000. Efficacy of nurse telehealth care and peer support in augmenting treatment of depression in primary care. Arch. Fam. Med. 9: 700-708. 